\title{
A Comparative Study Of The Methods Of Solving Non-Linear Programming Problem
}

\author{
Bimal Chandra Das \\ Department of Textile Engineering \\ Daffodil International University, Dhaka, Bangladesh \\ E-mail: bcdnar@yahoo.com.
}

\begin{abstract}
The work present in this paper is based on a comparative study of the methods of solving Non-linear programming (NLP) problem. We know that Kuhn-Tucker condition method is an efficient method of solving Non-linear programming problem. By using Kuhn-Tucker conditions the quadratic programming (QP) problem reduced to form of Linear programming $(L P)$ problem, so practically simplex type algorithm can be used to solve the quadratic programming problem (Wolfe's Algorithm).We have arranged the materials of this paper in following way. Fist we discuss about non-linear programming problems. In second step we discuss Kuhn- Tucker condition method of solving NLP problems. Finally we compare the solution obtained by Kuhn- Tucker condition method with other methods. For problem so consider we use MATLAB programming to graph the constraints for obtaining feasible region. Also we plot the objective functions for determining optimum points and compare the solution thus obtained with exact solutions.
\end{abstract}

Keywords: Non-linear programming, objective function, convex-region, pivotal element, optimal solution.

\section{Introduction}

For decision making optimization plays the central role. Optimization is the synonym of the word maximization/minimization. It means choose the best. In our time to take any decision, we use most modern scientific methods best on computer implementations. Modern optimization theory based on computing and we can select the best alternative value of the objective function. The optimization problems have two major divisions. One is linear programming problem and other is non-linear programming problem [1]. But the modern game theory, dynamic programming problem, integer programming problem also part of the optimization theory having wide range of application in modern science, economics and management. Linear and non-linear programming problem optimizes an objective function subject to a class of linear and nonlinear equality or inequality conditions called constraints, usually subject to non-negativity restrictions of the variables. It was introduced by [2]. In the present work we tried to compare some methods of non-linear programming problem. We know that, for solving a non-linear programming problem various algorithms can be used, but only few of the methods will be affective for solving problems. Usually none of the algorithms have no relations with others and there is no universal algorithm like simplex method in linear programming for solving a non-linear programming problem. In the work we will apply the methods for solving problem rather than its theoretical descriptions.

\section{Non-linear programming}

Like linear programming, non-linear programming is a mathematical technique for determining the optimal solutions to many business problems. The problem of maximizing or minimizing a given function

$$
\left.\begin{array}{l}
Z=f(\underline{x}) \\
\text { Subject to } \\
\quad g_{i}(\underline{x}) \leq b_{i}\left(\geq b_{i}\right)
\end{array}\right\}
$$

is called to general non-linear programming problem, if the objective function i.e. the function $f(x)$ or any one of the constraints function $g_{i}(\underline{x})$ is non-linear ; or both are non-linear for $x$ nonnegative. In a word, the non-linear programming problem is that of choosing nonnegative values of certain variables, so as to maximize or minimize a given non-linear function subject to a given set of linear or non-linear inequality constraints; or maximize or minimize a linear 
function subject to a given set of non-linear inequality. The problem (2.1) can be re written as:

$$
\begin{aligned}
& \text { Maximize } \mathrm{Z}=f\left(x_{1}, x_{2}, x_{3}, \ldots ., \mathrm{x}_{\mathrm{n}}\right) \\
& \text { Subject to } \\
& g_{1}\left(x_{1}, x_{2}, x_{3}, \ldots ., x_{n}\right) \leq b_{1}\left(\geq b_{1}\right) \\
& g_{2}\left(x_{1}, x_{2}, x_{3}, \ldots ., x_{n}\right) \leq b_{2}\left(\geq b_{2}\right) \\
& \left.\begin{array}{l}
g_{m}\left(x_{1}, x_{2}, x_{3}, \ldots, x_{n}\right) \leq b_{m}\left(\geq b_{m}\right) \\
x_{1} \geq 0, \quad x_{2} \geq 0, \quad \ldots x_{n} \geq 0
\end{array}\right\}
\end{aligned}
$$

Any vector $x$ satisfying the constraints \& non-negativity restrictions will be called a feasible solution for the problem. Geometrically, each of the n- no negativity restrictions $x_{j} \geq 0, \quad j=1,2,3 \ldots . . n$ defines a half- space of non-negative values \& the intersection of all such half-spaces is the nonnegative orthant, a subset of Euclidian nspace. In $E^{2}$ the non-negative orthant is the non-negative first quadrant. Each of the ninequality constraints and it was introduced by [3].

$$
g_{i}(x) \leq \mathrm{b}_{\mathrm{i}}\left(\geq \mathrm{b}_{\mathrm{i}}\right) \quad \mathrm{i}=1,2, \ldots, \mathrm{m}
$$

also defines a set of points in Euclidian nspace and the intersection of these m-sets with non-negative orthant is called as opportunity set i.e.

$\mathrm{X}=\left\{x \in \mathrm{E}^{\mathrm{n}}: \mathrm{g}(x) \leq \mathrm{b} ; x \geq 0\right\}$

So, geometrically a non-linear programming problem is that of finding a point or a set of points in the opportunity set at which the highest contour of the objective function is attained. We know that the optimum solution of linear programming problem does occur at an extreme point of the convex opportunity set. However in case of non-linear programming problem the solution can exist at the boundary or in the interior of the opportunity set. (Weierstrass theorem : If the non-empty set $\mathrm{X}$ is compact (i.e. closed and bounded ) and objective function $F(x)$ is continuous on $\mathrm{X}$ then $\mathrm{F}(x)$ has a global maximum either in $\mathrm{X}$ in the interior or on the boundary of $X^{2}$.[5])

\section{Kuhn-Tucker Theory}

The impetus for generalizations of this theory is contained in the material presented where we observed that under certain conditions, a point at which $f(x)$ takes on a relative maximum for points satisfying $g_{i}(x)=b_{i}, i=1,2, \ldots ., m$, is a saddle point of the Lagrangian function $\quad \mathrm{F}(x, \lambda)$. The material presented was originally developed By H.W. Kuhn and A.W.Tucker [5]. The theorem has been of fundamental importance in developing a numerical procedure for solving quadratic programming problems.

A function $F(x, \lambda), x$-being an n-component and $\lambda$ an m-component vector, is said to have a saddle point at $\left[x_{\circ}, \lambda_{\circ}\right]$ if

$$
F\left(x, \lambda_{\circ}\right) \leq F\left(x_{\circ}, \lambda_{\circ}\right) \leq F\left(x_{\circ}, \lambda\right)
$$

holds for all $x$ in an $\in$-neighborhood of $\lambda$. If (5) holds for all $x$ and $\lambda$, then $F(x, \lambda)$ is said have a saddle point in the large on a global saddle point at $\left(x_{\circ}, \lambda_{\circ}\right)$. We shall find it convenient to specialize the definition of a saddle point to cases where certain components of $\mathrm{x}$ and $\lambda$ are restricted to be non-negative, others are to be non-positive, and a third category is to be unrestricted in sign.

$$
\left.\begin{array}{l}
\frac{\partial}{\partial x_{j}} F\left(x_{\circ}, \lambda_{\circ}\right) \leq 0, \quad j=1, \ldots . ., s \\
\frac{\partial}{\partial x_{j}} F\left(x_{\circ}, \lambda_{\circ}\right) \geq 0, \quad j=s+1, \ldots . ., t \\
\frac{\partial}{\partial x_{j}} F\left(x_{\circ}, \lambda_{\circ}\right)=0, \quad j=t+1, \ldots . ., n
\end{array}\right\}
$$

$x_{j}{ }^{\circ} \geq 0, \quad j=1, \ldots ., s ; \quad x_{j}{ }^{\circ} \leq 0$,

$j=s+1, \ldots . ., t$;

$x_{j}^{\circ} \quad$ unrestricted, $j=t+1, \ldots . . n$;

$x_{j}^{\circ} \frac{\partial}{\partial x_{j}} F\left(x_{\circ}, \lambda_{\circ}\right)=0, j=1, \ldots . ., n$;

$\frac{\partial}{\partial \lambda_{i}} F\left(x_{\circ}, \lambda_{\circ}\right) \geq 0, \quad i=1, \ldots . ., u$

$\frac{\partial}{\partial \lambda_{i}} F\left(x_{\circ}, \lambda_{\circ}\right) \leq 0, i=u+1, \ldots . ., v$

$\frac{\partial}{\partial \lambda_{i}} F\left(x_{\circ}, \lambda_{\circ}\right)=0, \quad i=v+1, \ldots . ., m$

$\lambda_{i}^{\circ} \geq 0, i=1, \ldots, u ; \lambda_{i}^{\circ} \leq 0, i=u+1, \ldots, v$

$\lambda_{i}^{\circ}$ unrestricted, $i=v+1, \ldots, m$

$\lambda_{i}^{\circ} \frac{\partial}{\partial \lambda_{i}} F\left(x_{\circ}, \lambda_{\circ}\right)=0, \quad i=1, \ldots ., m$. 
Equations (6) through (12) represent a set of necessary conditions which $\left[x_{\circ}, \lambda_{\circ}\right]$ must satisfy if $F(x, \lambda)$ has a saddle point at $\left[x_{\circ}, \lambda_{\circ}\right]$ for $[x, \lambda] \in W$, provided that $F \in c^{\prime}$.

Now the sufficient conditions can be stated as follows. Let $\left[x_{\circ}, \lambda_{\circ}\right]$ be a point satisfying (6) through (12). Then if there exists an $\in$ neighborhood about $\left[x_{0}, \lambda_{\circ}\right]$ such that for points $\left[x, \lambda_{0}\right] \in W$, in this neighborhood $F\left(x, \lambda_{\circ}\right) \leq F\left(x_{\circ}, \lambda_{\circ}\right)+\nabla_{x} F\left(x_{\circ}, \lambda_{\circ}\right)\left(x-x_{\circ}\right)$ $F\left(x_{\circ}, \lambda\right) \geq F\left(x_{\circ}, \lambda_{\circ}\right)+\nabla_{\lambda} F\left(x_{\circ}, \lambda_{\circ}\right)\left(\lambda-\lambda_{\circ}\right)$ then $F(x, \lambda)$ has a saddle point at $\left[x_{0}, \lambda_{\circ}\right]$ for $[x, \lambda] \in W$. If (13) and (14) hold for all $x \in W_{1}, \lambda \in W_{2}$, it follows that has a global saddle point at $\left[x_{\circ}, \lambda_{\circ}\right]$ for $[x, \lambda] \in W$.

From the above we can deduce KuhnTucker's conditions as follows:

Let us consider the non-linear programming problem as:

$$
\begin{array}{ll}
\text { Maximize } Z=f(x) \\
\text { subject to } & g(x) \leq b \\
& x \geq 0 .
\end{array}
$$

To obtain Kuhn-Tucker necessary conditions, let us convert the inequality constrains of the above problem to equality constraints by adding a vector of $\mathrm{m}$ slack (surplus) variables:

$$
\operatorname{Max} Z=f(x)
$$

subject to $g(x)+s=b$

or $s=b-g(x)$ where $s^{T}=\left(s_{1}, s_{2}, \ldots . ., s_{m}\right)$. Now the Lagrangian function for the problem takes the form, $L(x, \lambda)=f(x)+\lambda\{b-g(x)\}$

Assumed that $f, g \in c^{\prime}$, first order necessary conditions can be obtained by taking first order derivatives with respect to $x, \lambda$ of the Lagrangian function

$$
\begin{array}{ll} 
& \frac{\partial F}{\partial x} \equiv \frac{\partial f}{\partial x}-\lambda \frac{\partial g}{\partial x} \leq 0 \\
\therefore \quad & \frac{\partial F}{\partial x} x \equiv\left(\frac{\partial f}{\partial x}-\frac{\partial g}{\partial x}\right) x=0 \\
\text { and } \quad & \frac{\partial F}{\partial \lambda} \equiv b-g(x) \geq 0 \\
\therefore \quad & \lambda \frac{\partial F}{\partial \lambda} \equiv \lambda(b-g(x))=0 .
\end{array}
$$

Rewriting the Lagrangian form we get
$F(x, \lambda) \equiv f(x)+\sum_{i} \lambda_{i}\left(b_{i}-g_{i}(x)\right), i=1, \ldots . ., m$.

Now if $f(x)$ takes constraints local optimum at $x=x^{*}$ it is necessary that a vector $\lambda^{*}$ exists such that

$$
\begin{aligned}
& 1 . \nabla_{x} F\left(x^{*}, \lambda^{*}\right) \equiv \nabla_{x}\left(f\left(x^{*}\right)\right)-\sum_{i} \lambda_{i}^{*} \nabla_{x} g_{i}\left(x^{*}\right) \leq 0 \\
& \text { 2. }\left[\nabla_{x} F\left(x^{*}, \lambda^{*}\right)\right]^{T} x^{*} \equiv \sum_{j=1}^{n}\left[\nabla_{x}\left(f\left(x^{*}\right)\right)\right. \\
& \left.-\sum_{i} \lambda_{i}{ }^{*} \nabla_{x} g_{i}\left(x^{*}\right) \leq 0\right] x_{j}^{*}=0 \\
& \text { 3. } \nabla_{\lambda} F\left(x^{*}, \lambda^{*}\right) \equiv b_{i}-g_{i}\left(x^{*}\right) \geq 0 \text { if } g_{i}\left(x^{*}\right) \leq b_{i} \\
& \equiv b_{i}-g_{i}\left(x^{*}\right) \leq 0 \quad \text { if } \quad g_{i}\left(x^{*}\right) \geq b_{i} \\
& \equiv b_{i}-g_{i}\left(x^{*}\right)=0 \quad \text { if } \quad g_{i}\left(x^{*}\right)=b_{i} \\
& \text { 4. }\left[\nabla_{\lambda} F\left(x^{*}, \lambda^{*}\right)\right] \lambda^{*} \equiv \sum_{i}\left[b_{i}-g_{i}\left(g^{*}\right)\right] \lambda_{i}^{*}=0 \text {. }
\end{aligned}
$$

\section{Comparison of solutions by Kuhn-} Tucker condition and others

In this part we want to show that various NLP problems can be solved by different methods. Our aim is to show the affective ness of the methods considered:

Example: Let us consider the problem

Maximize $Z=2 x_{1}+3 x_{2}-x_{1}{ }^{2}$

Subject to the constraints:

$$
\begin{aligned}
& x_{1}+2 x_{2} \leq 4 \\
& x_{1}, x_{2} \geq 0
\end{aligned}
$$

First we want to solve above problem by graphical solution method.

The given problem can be rewriting as:

Maximize $Z=-\left(x_{1}-1\right)^{2}+3\left(x_{2}+\frac{1}{3}\right)$

Subject to the constraints

$$
\begin{aligned}
& x_{1}+2 x_{2} \leq 4 \\
& x_{1}, x_{2} \geq 0
\end{aligned}
$$

We observe that our objective function is a parabola with vertex at $(1,-1 / 3)$ and constraints are linear. To solve the problem graphically, first we constract the graph of the constraint in the first quadrant since $x_{1} \geq 0$ and $\quad x_{2} \geq 0$ by considering the inequation to equation. $\quad x_{1}+2 x_{2}=4$ and it was introduced by [6].

Each point has co-ordinates of the tupe $\left(x_{1}, x_{2}\right)$

and conversely every ordered pair $\left(x_{1}, x_{2}\right)$ or real numbers determines a point in the plane. Thus our search for the number pair 
$\left(x_{1}, x_{2}\right)$ is restricted to the points of the first quadrant only

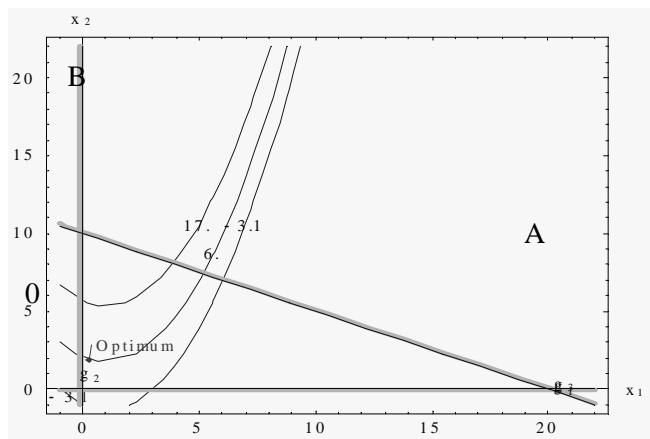

Fig. 1 Optimum solution by graphical method

We get the convex region $\mathrm{OAB}$ as opportunity set. Since our search is for such a pair $\left(x_{1}, x_{2}\right)$ which gives a maximum value of $2 x_{1}+3 x_{2}-x_{1}^{2}$ and lies in the convex region. The desire point will be that point of the region at which a side of the convex region is tangent to the parabola. For this proceed as follows:

Differentiating the equation of the parabola, we get

$$
\begin{aligned}
& 2 d x_{1}+3 d x_{2}-2 x_{1} d x_{1}=0 \\
& \Rightarrow \frac{d x_{2}}{d x_{1}}=-\frac{2-2 x_{1}}{3}
\end{aligned}
$$

Now differentiating the equation of the constraint, we get

$$
d x_{1}+2 d x_{2}=0 \Rightarrow \frac{d x_{2}}{d x_{1}}=-\frac{1}{2}
$$

Solving equation (15) and (16), we get $x_{1}=\frac{1}{4}$ and $x_{2}=\frac{15}{8}$. This shows that the parabola $z=-\left(x_{1}-1\right)^{2}+3\left(x_{2}+\frac{1}{3}\right)$ has a tangent to it the line $x_{1}+2 x_{2}=4$ at the point $\left(\frac{1}{4}, \frac{15}{8}\right)$. Hence we get the maximum value of the objective function at this point. Therefore,

$$
\begin{aligned}
\mathrm{Z}_{\max } & =2 x_{1}+3 x_{2}-x_{1}^{2} \\
& =\frac{97}{16} \quad \text { at } x_{1}=\frac{1}{4}, \mathrm{x}_{2}=\frac{15}{8} .
\end{aligned}
$$

Let us solve the above problem by using [7] Kuhn-Tucker Conditions. The Lagrangian function of the given problem is
$F\left(x_{1}, x_{2}, \lambda\right) \equiv 2 x_{1}+3 x_{2}-x_{1}^{2}+\lambda\left(4-x_{1}-2 x_{2}\right)$.

By Kuhn-Tucker conditions, we obtain

(a) $\frac{\partial F}{\partial x_{1}} \equiv 2-2 x_{1}-\lambda \leq 0, \quad \frac{\partial F}{\partial x_{2}} \equiv 3-2 \lambda \leq 0$

(b) $\frac{\partial \mathrm{F}}{\partial \lambda} \equiv 4-x_{1}-2 x_{2} \geq 0$

(c) $x_{1} \frac{\partial F}{\partial x_{1}}+x_{2} \frac{\partial F}{\partial x_{2}} \equiv x_{1}\left(2-2 x_{1}-\lambda\right)+x_{2}(3-2 \lambda)=0$

(d) $\lambda \frac{\partial \mathrm{F}}{\partial \lambda} \equiv \lambda\left(4-x_{1}-2 x_{2}\right)=0$ with $\lambda \geq 0$.

Now there arise the following cases:

Case (i) : Let $\lambda=0$, in this case we get from

$\frac{\partial F}{\partial x_{1}} \equiv 2-2 x_{1} \leq 0$ and $\frac{\partial F}{\partial x_{2}} \equiv 3-2 \cdot 0 \leq 0$

$\Rightarrow 3 \leq 0$ which is impossible and this solution is to be discarded and it was introduced by [8].

Case (ii): Let $\lambda \neq 0$. In this case we get from

$\lambda\left(4-x_{1}-2 x_{2}\right)=0$

$4-x_{1}-2 x_{2}=0 \Rightarrow x_{1}+2 x_{2}=4$

Also from $\frac{\partial F}{\partial x_{1}} \equiv 2-2 x_{1}-\lambda \leq 0$

$$
\begin{gathered}
\frac{\partial F}{\partial x_{2}} \equiv 3-2 \lambda \leq 0 \quad \therefore 2 x_{1}+\lambda-2 \geq 0 \\
\text { and } 2 \lambda-3 \geq 0 \Rightarrow \lambda \geq \frac{3}{2}
\end{gathered}
$$

If we take $\lambda=\frac{3}{2}$, then $2 x_{1} \geq \frac{1}{2}$

If we consider $2 x_{1}=\frac{1}{2}$ then $x_{1}=\frac{1}{4}$.

Now putting the value of $x_{1}$ in (4 .3),

we get $\mathrm{x}_{2}=\frac{15}{8}$

$\therefore\left(x_{1}, x_{2}, \lambda\right)=\left(\frac{1}{4}, \frac{15}{8}, \frac{3}{2}\right)$. for this solution

$\frac{\partial F}{\partial x_{1}} \equiv 2-2 \cdot \frac{1}{4}-\frac{3}{2}=\frac{4-1-3}{2}=0$ satisfied

$\frac{\partial \mathrm{F}}{\partial x_{2}} \equiv 3-2 \cdot \frac{3}{2}=0 \quad$ satisfied

$\frac{\partial \mathrm{F}}{\partial \lambda} \equiv 4-\frac{1}{4}-2 \cdot \frac{15}{8_{4}}=\frac{16-1-15}{4}=0$ satisfied

$\mathrm{x}_{1} \frac{\partial F}{\partial x_{1}}+x_{2} \frac{\partial F}{\partial x_{2}} \equiv \frac{1}{4} \times 0+\frac{15}{8} \times 0=0 \quad$ satisfied

$\lambda \frac{\partial \mathrm{F}}{\partial \lambda} \equiv \frac{3}{2} \cdot 0=0 \quad$ satisfied

Thus all the Kuhn-Tucker necessary conditions are satisfied at the point $\left(\frac{1}{4}, \frac{15}{8}\right)$. 
Hence the optimum (maximum) solution to the given NLP problem is

$$
\begin{aligned}
Z_{\max } & =2 x_{1}+3 x_{2}-x_{1}^{2} \\
& =2 \cdot \frac{1}{4}+3 \cdot \frac{15}{8}-\left(\frac{1}{4}\right)^{2} \\
& =\frac{97}{16} \text { at } x_{1}=\frac{1}{4}, x_{2}=\frac{15}{8} .
\end{aligned}
$$

Let us solve the problem by Beale's method.

Maximize $f(x)=2 x_{1}+3 x_{2}-x_{1}^{2}$

Subject to the constraints:

$$
\begin{aligned}
& x_{1}+2 x_{2} \leq 4 \\
& x_{1}, x_{2} \geq 0
\end{aligned}
$$

Introducing a slack variables $s$, the constraint becomes

$$
\begin{aligned}
& x_{1}+2 x_{2}+s=4 \\
& x_{1}, x_{2} \geq 0
\end{aligned}
$$

since there is only one constraint, let $\mathrm{s}$ be a basic variable. Thus we have by [9]

$x_{B}=(s), x_{N B}=\left(x_{1}, x_{2}\right)$ with $s=4$

Expressing the basic $x_{B}$ and the objective function in terms of non-basic $x_{N B}$, we have $\mathrm{s}=4-\mathrm{x}_{1}-2 \mathrm{x}_{2}$ and $\mathrm{f}=2 \mathrm{x}_{1}+3 \mathrm{x}_{2}-\mathrm{x}_{1}{ }^{2}$.

We evaluated the partial derivatives of $f$ w.r.to non-basic variables at $x_{N B}=0$, we get

$$
\begin{aligned}
& \left(\frac{\partial f}{\partial x_{1}}\right)_{x_{N B=0}}=\left(2-2 x_{1}\right)_{x_{N B=0}}=2-2 \cdot 0=2 \\
& \left(\frac{\partial f}{\partial x_{2}}\right)_{x_{N B=0}}=3
\end{aligned}
$$

since both the partial derivatives are positive, the current solution can be improved. As $\frac{\partial f}{\partial x_{2}}$ gives the most positive value, $x_{2}$ will enter the basis.

Now, to determine the leaving basic variable, we compute the ratios:

$$
\begin{aligned}
\min \left\{\frac{\alpha_{h o}}{\left|\alpha_{h k}\right|}, \frac{\gamma_{k o}}{\gamma_{k k}}\right\} & =\min \left\{\frac{\alpha_{30}}{\left|\alpha_{32}\right|}, \frac{\gamma_{20}}{\left|\alpha_{22}\right|}\right\} \\
& =\min \left\{\frac{4}{|-2|}, \frac{3}{0}\right\}=2
\end{aligned}
$$

since the minimum occurs for $\frac{\alpha_{30}}{\left|\alpha_{30}\right|}$, s will leave the basis and it was introduced by [10]. Thus expressing the new basic variable, $x_{2}$ as well as the objective function $f$ in terms of the new non-basic variables $\left(x_{1}\right.$ and $s$ ) we have:

$$
\text { and } \begin{aligned}
f & =2 x_{1}+3\left(2-\frac{x_{1}}{2}-\frac{x_{1}}{2}\right)-x_{1}^{2} \\
& =6+\frac{x_{1}}{2}-\frac{3}{2} s-x_{1}{ }^{2}
\end{aligned}
$$

we, again, evaluate the partial derivates of $f$ w. r. to the non-basic variables:

$\left(\frac{\partial f}{\partial x_{1}}\right)_{x_{N B}=0}=\left(\frac{1}{2}-2 x_{1}\right)_{x_{1=0}}=\frac{1}{2}$

$\left(\frac{\partial f}{\partial s}\right)_{x_{N B=0}}=-\frac{3}{2}$.

since the partial derivatives are not all negative, the current solution is not optimal, clearly, $x_{1}$ will enter the basis.

For the next Criterion, we compute the ratios $\min \left\{\frac{\alpha_{20}}{\left|\alpha_{21}\right|}, \frac{\gamma_{10}}{\left|\gamma_{11}\right|}\right\}=\left\{\frac{2}{|-1 / 2|}, \frac{1 / 2}{|-2|}\right\}=\frac{3}{4}$.

since the minimum of these ratios correspond to $\frac{\left|\gamma_{10}\right|}{\left|\gamma_{11}\right|}$, non-basic variables can be removed. Thus we introduce a free variable, $u_{1}$ (unrestricted), as an additional non-basic variable, defined by

$u_{1}=\frac{1}{2} \frac{\partial f}{\partial x_{1}}=\frac{1}{2}\left(\frac{1}{2}-2 x_{1}\right)=\frac{1}{4}-x_{1}$

Note that now the basis has two basic variables $x_{2}$ and $x_{1}$ (just entered). That is, we have

$x_{N B}=\left(s, u_{1}\right)$ and $x_{B}=\left(x_{1}, x_{2}\right)$

Expressing the basic $x_{B}$ in terms of non-basic $x_{N B}$, we have, $x_{1}=\frac{1}{4}-u_{1}$

and $x_{2}=\frac{1}{2}\left(4-x_{1}-x_{3}\right)=\frac{15}{8}+\frac{1}{2} u_{1}-\frac{1}{2} s$.

The objective function, expressing in terms of $x_{N B}$ is,

$$
\begin{aligned}
f & =2\left(\frac{1}{4}-u_{1}\right)+3\left(\frac{15}{8}+\frac{1}{2} u_{1}-\frac{1}{2} s\right)-\left(\frac{1}{4}-u_{1}\right)^{2} \\
& =\frac{97}{16}-\frac{3}{2} s-u_{1}^{2} .
\end{aligned}
$$

Now, $\left(\frac{\partial F}{\partial s}\right)_{\substack{x_{N B=0} \\ u=0}}=-\frac{3}{2} ;\left(\frac{\delta f}{\delta u_{1}}\right)_{\substack{X_{N B}=0 \\ u=0}}=-2 u_{1}=0$ since $\frac{\partial f}{\partial u_{1}} \leq 0$ for all $x_{j}$ in $x_{N B}$ and $\frac{\partial f}{\partial u}=0$, 
the current solution is optimal. Hence the optimal basic feasible solution to the given problem is:

$x_{1}=\frac{1}{4}, \quad x_{2} \frac{15}{8}, Z *=\frac{97}{16}$

Let us solve the given problem by [3] using Wolfe's algorithm

$\operatorname{Max} Z=2 x_{1}+3 x_{2}-x_{1}^{2}$

subject to the constraints

$$
\begin{aligned}
x_{1}+2 x_{2} & \leq 4 \\
x_{1}, x_{2} & \geq 0
\end{aligned}
$$

First we convert the inequality constraints to equality by introducing slack variables $\mathrm{s} \geq 0$ $x_{1}+2 x_{2}+s=4$

Therefore, the Lagrangian function of the problem is

$$
\begin{aligned}
& L(x, \lambda) \equiv 2 x_{1}+3 x_{2}-x_{1}^{2}+\lambda\left(4-x_{1}-2 x_{2}\right) \\
& \text { Here } \mathrm{D}=\left(\begin{array}{ll}
-1 & 0 \\
0 & 0
\end{array}\right), A=\left(\begin{array}{ll}
1 & 2
\end{array}\right), \mathrm{A}^{\mathrm{T}}=\left(\begin{array}{l}
1 \\
2
\end{array}\right), \\
& \mathrm{C}=\left(\begin{array}{l}
2 \\
3
\end{array}\right), \mathrm{b}=(4)
\end{aligned}
$$

We know by Wolfe's algorithm:

$$
\begin{gathered}
2 D X-A^{T} \lambda+V=-C \\
\Rightarrow 2\left(\begin{array}{rr}
-1 & 0 \\
0 & 0
\end{array}\right)\left(\begin{array}{l}
x_{1} \\
x_{2}
\end{array}\right)-\left(\begin{array}{l}
1 \\
2
\end{array}\right) \lambda+\left(\begin{array}{l}
v_{1} \\
v_{2}
\end{array}\right)=-\left(\begin{array}{l}
2 \\
3
\end{array}\right) \\
\Rightarrow-2 x_{1}-\lambda+v_{1}=-2 \\
-2 \lambda+v_{2}=-3 \\
\Rightarrow 2 x_{1}+\lambda-v_{1}=2 \\
2 \lambda-v_{2}=3
\end{gathered}
$$

Introducing two artificial variables $a_{1}$ and $a_{2}$ in equations (4. 5) and (4. 6), we get

$$
\begin{aligned}
& 2 x_{1}+\lambda-v_{1}+a_{1}=2 \\
& 2 \lambda-v_{2}+a_{2}=3
\end{aligned}
$$

The quadratic programming problem is equivalent to

$$
\operatorname{Min} Z=a_{1}+a_{2}
$$

i.e. $\operatorname{Max}(-Z)=-a_{1}-a_{2}$

subject to the constraints

$$
\begin{aligned}
& x_{1}+2 x_{2}+s=4 \\
& 2 x_{1}+\lambda-v_{1}+a_{1}=2 \\
& 2 \lambda-v_{2}+a_{2}=3 \\
& x_{1}, x_{2}, \lambda, v_{1}, v_{2}, s, a_{1}, a_{2} \geq 0 \\
& \text { with } x_{1} v_{1}=0, x_{2} v_{2}=0, \lambda s=0
\end{aligned}
$$

The solution of the problem can be shown in the following modified simplex tableau: It was Introduced by [10]. Here we get three constraints equations with eight unknown.
So, for basic solution of the system always three variables will take non-zero values and others are zero. We will find out non zero values for $x_{1}, x_{2} \& \lambda$ or $s$ In our case initial basic solution is $a_{1}=2, a_{2}=3, s=4 \Rightarrow z=0$. Our next goal is to improve the value of $\mathrm{Z}$. In tableau -1 , we put constraints system as:

Table 1 Constraints system

\begin{tabular}{|c|c|c|c|c|c|c|c|c|}
\hline$x_{1}$ & $x_{2}$ & $\lambda$ & $v_{1}$ & $v_{2}$ & $s$ & $a_{1}$ & $a_{2}$ & $c$ \\
\hline 1 & 2 & 0 & 0 & 0 & 1 & 0 & 0 & 4 \\
2 & 0 & 1 & -1 & 0 & 0 & 1 & 0 & 2 \\
0 & 0 & 2 & 0 & -1 & 0 & 0 & 1 & 3 \\
\hline
\end{tabular}

Now taking $x_{1}$ as leading variables. Then we get $\min (4 / 1,2 / 2)$ is $2 / 2$. So, second element of the $1^{\text {st }}$ column is the pivotal element and the corresponding column is the pivotal column. So, $x_{1}$ enters in basis. Reducing the pivotal element to unity by dividing all elements of the pivotal row by 2 , we get the table 2.

Table 2 Reducing first pivotal element to unity.

\begin{tabular}{|l|l|l|l|l|l|l|l|l|}
\hline$x_{1}$ & $x_{2}$ & $\lambda$ & $v_{1}$ & $v_{2}$ & $s$ & $a_{1}$ & $a_{2}$ & $c$ \\
\hline 1 & 2 & 0 & 0 & 0 & 1 & 0 & 0 & 4 \\
1 & 0 & $1 / 2$ & $-1 / 2$ & 0 & 0 & $1 / 2$ & 0 & 1 \\
0 & 0 & 2 & 0 & -1 & 0 & 0 & 1 & 3 \\
\hline
\end{tabular}

Reducing zero all elements of the pivotal column except pivotal one, we get the table 3

Table 3 Reducing zero all elements of 1st pivotal column except pivotal one.

\begin{tabular}{|c|c|c|c|c|c|c|c|c|}
\hline $\mathrm{x}_{1}$ & $\mathrm{x}_{2}$ & $\lambda$ & $\mathrm{v}_{1}$ & $\mathrm{v}_{2}$ & $\mathrm{~s}$ & $\mathrm{a}_{1}$ & $\mathrm{a}_{2}$ & $\mathrm{c}$ \\
\hline 0 & 2 & $-1 / 2$ & $1 / 2$ & 0 & 1 & $-1 / 2$ & 0 & 3 \\
1 & 0 & $1 / 2$ & $-1 / 2$ & 0 & 0 & $1 / 2$ & 0 & 1 \\
0 & 0 & 2 & 0 & -1 & 0 & 0 & 1 & 3 \\
\hline
\end{tabular}

Now taking $x_{2}$ as our next leading variable. Then we get 1st element of the second column is our next pivotal element. Reducing it to unity by dividing all elements of the pivotal now by 2 and next taking $\lambda$ as our next leading variable. Then we get $\min \left\{\frac{1}{1 / 2}, \frac{3}{2}\right\}$ is $\frac{3}{2}$. So, 2 is our next pivotal element. Reducing the pivotal element to unity, we get, the tableau-5.

Table 4 Reducing $3{ }^{\text {rd }}$ pivotal element to unity.

\begin{tabular}{|c|c|c|c|c|c|c|c|c|}
\hline $\mathrm{x}_{1}$ & $\mathrm{x}_{2}$ & $\lambda$ & $\mathrm{v}_{1}$ & $\mathrm{v}_{2}$ & $\mathrm{~s}$ & $\mathrm{a}_{1}$ & $\mathrm{a}_{2}$ & $\mathrm{c}$ \\
\hline 0 & 1 & $-1 / 4$ & $1 / 4$ & 0 & $1 / 2$ & $-1 / 4$ & 0 & $3 / 2$ \\
1 & 0 & $1 / 2$ & $-1 / 2$ & 0 & 0 & $1 / 2$ & 0 & 1 \\
0 & 0 & 1 & 0 & $-1 / 2$ & 0 & 0 & $1 / 2$ & $3 / 2$ \\
\hline
\end{tabular}


Making zero all elements of the pivotal column except pivotal one, we get the table 5 .

Table 5 Optimal solution

\begin{tabular}{|l|c|c|c|c|c|c|c|c|}
\hline $\mathrm{x}_{1}$ & $\mathrm{x}_{2}$ & $\lambda$ & $\mathrm{V}_{1}$ & $\mathrm{~V}_{2}$ & $\mathrm{~S}$ & $\mathrm{a}_{1}$ & $\mathrm{a}_{2}$ & $\mathrm{C}$ \\
\hline 0 & 1 & 0 & $1 / 4$ & $-1 / 8$ & $1 / 2$ & $-1 / 4$ & $1 / 8$ & $15 / 8$ \\
1 & 0 & 0 & $-1 / 2$ & $1 / 4$ & 0 & 0 & $-1 / 4$ & $1 / 4$ \\
0 & 0 & 1 & 0 & $-1 / 2$ & 0 & 0 & $1 / 2$ & $3 / 2$ \\
\hline
\end{tabular}

From T-5 we obtain the optimal solution as $x_{1}=\frac{1}{4}, x_{2}=\frac{15}{8}, \lambda=\frac{3}{2}$.

Thus for the optimal solution for the given QP problem is

$$
\begin{aligned}
\operatorname{Max} & Z=2 x_{1}+3 x_{2}-x_{1}{ }^{2} \\
& =2 \cdot \frac{1}{4}+3 \cdot \frac{15}{8}-\left(\frac{1}{4}\right)^{2} \\
& =\frac{97}{16} \text { at }\left(x_{1}{ }^{*}, x_{2}{ }^{*}\right)=\left(\frac{1}{4}, \frac{15}{8}\right) .
\end{aligned}
$$

which is same as we obtain by Kuhn-Tuker condition method. For all kinds of non-linear programming problem, we can show that the optimal solution by Kuhn-tucker condition is same as any other method we considered Therefore the solution obtained by graphical solution method, Kuhn-Tucker conditions and Wolfe's method are same.

\section{Conclusion}

To obtain an optimal solution to the nonlinear programming problem, we observe that Kuhn-Tucker conditions are more useful than any other methods of solving NLP problem. Because in a NLP problem particular problems are solve by particular method. There is no universalism in the methods of solving NLP problem. But we have shown that by Kuhn-Tucker conditions all kinds of NLP problems can be solved. The NLP problem involving two variables can easily solve by graphical solution method, but the problem involving more variables cannot solve by graphical solution method. Besides, for all NLP problems, graphical solution method do not gives always-optimal solution. Only quadratic programming problems can solve by Wolfe's method.

Therefore, from the above discussion, we can say that kuhn-Tucker conditions are the best method for solving only NLP problem.

\section{References}

[1] Greig, D. M: “Optimization”. Lougman- Group United, New York (1980).

[2] Gupta, P. K. Man Mohan: "Linear programming and theory of Games" Sultan Chand \& sons, New Delhi, India

[3] Honhendalken, B. Von: "Simplicial Decomposition in Non-linear Programming procedure".

[4] G. Hadley: "Non-linear and dynamic programming".

[5] M. S. Bazaraa \& C. M. Shetty: "Non-linear programming theory and algorithms".

[6] Mittal Sethi: "Linear Programming "'Pragati Prakashan, Meerut India 1997.

[7] Adadie. J: "On the Kuhn-Tucker Theory," in NonLinear Programming, J. Adadie. (Ed) 1967b.

[8] Hildreth. C: "A Quadratic Programming procedure”. Naval Research Logistics Quarterly.

[9] D. M.Himmeldlau: Applied Non-linear programming.

[10] G. R. Walsh: "Methods of optimization” Jon Wiley and sons ltd, 1975, Rev. 1985.

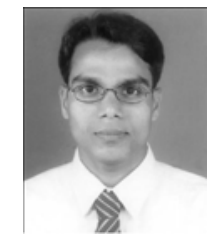

Bimal Chandra Das has completed M. Sc in pure Mathematics and B. Sc (Hons) in Mathematics from Chittagong University. Now he is working as a Lecturer under the Department of Textile Engineering in Daffodil International University. His area of research is Operation Research. 